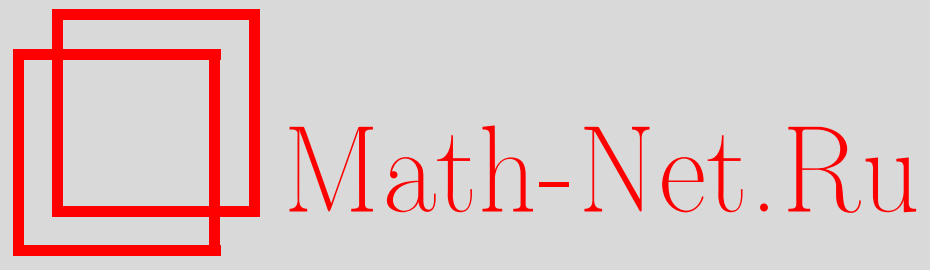

V. S. Korolyuk, N. Limnios, Poisson approximation of increment processes with Markov switching, Теория вероятн. и ее примен., 2004, том 49, выпуск 4, 726-742

DOI: https://doi.org/10.4213/tvp191

Использование Общероссийского математического портала Math-Net.Ru подразумевает, что вы прочитали и согласны с пользовательским соглашением

http://www. mathnet.ru/rus/agreement

Параметры загрузки:

IP: 18.208 .226 .222

26 апреля 2023 г., 13:42:34

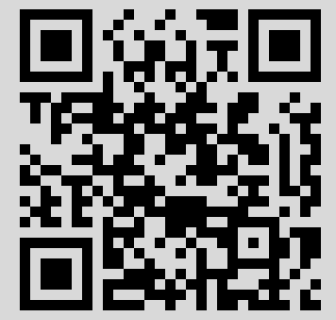




\section{POISSON APPROXIMATION OF INCREMENT PROCESSES WITH MARKOV SWITCHING}

Приводятся результаты о слабой сходимости процессов приращений с марковскими переключениями к составному процессу Пуассона.

Рассматривается также марковский процесс переключений с асимптотическим расшепленным фазовым пространством.

Эти результаты получены методами теории семимартингалов.

Ключевые слова и фразы: процесс приращений, семимартингал, марковский процесс. слабая сходимость, составной процесс Пуассона.

1. Introduction. Consider a sequence of random variables (r.v.'s) $\alpha_{k}$, $k \geqslant 0$, and a multivariate point process $\tau_{k}, x_{k}, k \geqslant 1$, (see [1], [3], [7], [9], [13]) with counting process $\nu(t):=\sup \left\{k \geqslant 1: \tau_{k} \leqslant t\right\}$. The stochastic process $A(t), t \geqslant 0$, defined by

$$
A(t):=\sum_{k=1}^{\nu(t)} \alpha_{k}\left(\theta_{k}, x_{k-1}\right)
$$

is called an increment process [3], [7]. We consider increment process with Markov switching as an additive semimartingale [4]. If the r.v.'s $\alpha_{k}, k \geqslant 1$, are independent and identically distributed (i.i.d.) and the multivariate point process $\tau_{k}, x_{k}, k \geqslant 0, \theta_{k+1}=\tau_{k+1}-\tau_{k}$, is just a renewal point process on $\mathbf{R}_{+}$, then (1) is called a compound process or a renewal reward process [15]. If the r.v.'s $\alpha_{k}, k \geqslant 0$, are i.i.d. and the multivariate point process $\tau_{k}, x_{k}, k \geqslant 0$, is just a Poisson point process on $\mathbf{R}_{+}$, then (1) is called a compound Poisson process [5]. If $\alpha_{k}$ is a fixed function defined on $\mathbf{R} \times E$, then (1) is a shot noise process [16], which plays an important role in the theory of noise of physical devices. In [8] the authors consider the random measure $\alpha_{k}\left(\tau_{k}\right)$ and a Poisson process $\nu(t)$, and they derive asymptotic results for (1) with application to insurance. For a semimartingale representation see [3], [4], $[7],[13],[14]$.

* Ukrainian National Academy of Science, Ukraine; e-mail: korol@imath.kiev.ua

** Université de Technologie de Compiègne, France; e-mail: Nicolaos.limnios@utc.fr 
This kind of processes are widely used in applications, i.e., risk and storage theory [17], reliability and maintenance theory [15], finance and insurance [8], noise of physical device [16], etc. In applications, $\theta_{k+1}$ is the acting time of the $k$-th event and $\alpha_{k}$ its magnitude or cost, etc.

In many applied problems the r.v.'s $\alpha_{k}$ depend on the environment. For example, the cost of a damage for an insurance company depends on which place, time, weather, etc., where it happens. In the case where we have a multistate environment, $E$ say, we suppose that the r.v.'s $\alpha_{k}$ depend on the state $x \in E: \alpha_{k}=\alpha_{k}(x)$.

The increment process considered here is based on a multivariate point process which corresponds to a Markov renewal representation of a Markov process and the r.v.'s $\alpha_{k}$ depend on the states of that Markov process. The convergence of the increment process towards a compound Poisson process is due to the fact that we assume the r.v.'s $\alpha_{k}$ take small values with big probabilities and big values with small probabilities.

This work is a continuation of previous works by the authors [10]-[12] on integral functionals of Markov processes. In fact, an integral functional can be represented also by the sum of an increment process and of another term, i.e.,

$$
\int_{0}^{t} a(x(s)) d s=\sum_{k=1}^{\nu(t)} a\left(x_{k-1}\right) \theta_{k}+a\left(x_{\nu(t)}\right)\left(t-\tau_{\nu(t)}\right),
$$

where $x(t), t \geqslant 0$, is a Markov (or a semi-Markov) jump process, $x_{k}, k \geqslant 0$, is its embedded Markov chain and $\theta_{k}$ are the interarrivals jump times.

In Section 2 we give a more precise mathematical definition of the increment process that we consider here. In Section 3 we give results on weak convergence of the increment processes towards a compound Poisson process, and in Section 4 we give weak convergence results with asymptotic split of phase state space of the underlying Markov process. Finally, in Section 5 we give proofs of theorems.

2. Preliminaries. Let $x(t), t \geqslant 0$, be a Markov jump process on a Polish space $(E, \mathscr{E})$ defined by the generator

$$
Q \varphi(x)=q(x) \int_{E} P(x, d y)[\varphi(y)-\varphi(x)]
$$

The semi-Markov kernel

$$
Q(x, B, t)=P(x, B)\left(1-e^{-q(x) t}\right), \quad x \in E, \quad B \in \mathscr{E}, \quad t \geqslant 0,
$$

defines the associated Markov renewal process $x_{k}, \tau_{k}, k \geqslant 0$, where $x_{k}, k \geqslant 0$, is the embedded Markov chain defined by the stochastic kernel

$$
P(x, B)=\mathbf{P}\left(x_{k+1} \in B \mid x_{k}=x\right)
$$


and $\tau_{k}, k \geqslant 0$, is the point process of jump times defined by the distribution function of sojourn times $\theta_{k+1}=\tau_{k+1}-\tau_{k}, k \geqslant 0$,

$$
\mathbf{P}\left(\theta_{k+1} \leqslant x \mid x_{k}=x\right)=1-e^{-q(x) t} .
$$

We suppose that the Markov process $x(t), t \geqslant 0$, is uniformly ergodic with stationary distribution $\pi(B), B \in \mathscr{E}$. Thus the embedded Markov chain $x_{k}$, $k \geqslant 0$, is uniformly ergodic too, with stationary distribution $\rho(B), B \in \mathscr{E}$, connected with $\pi$ by the following relation:

$$
\pi(d x) q(x)=q \rho(d x), \quad q:=\int_{E} \pi(d x) q(x) .
$$

In the sequel we will suppose that

$$
0<q_{0} \leqslant q(x) \leqslant q_{1}<+\infty, \quad x \in E .
$$

The increment process with Markov switching is defined by the sum

$$
A_{\varepsilon}(t):=\sum_{k=1}^{\nu(t / \varepsilon)} \alpha_{k}^{\varepsilon}\left(x_{k}\right), \quad t \geqslant 0,
$$

where $\nu(t)=\max \left\{k: \tau_{k} \leqslant t\right\}$ is the counting process of jumps. The family of r.v.'s $\alpha_{k}^{\varepsilon}(x), k \geqslant 1, x \in E$, is considered in the series scheme with a small series parameter $\varepsilon>0$, and is defined by the following distribution functions on the real line $\mathbf{R}$ :

$$
\Phi_{x}^{\varepsilon}(u)=\mathbf{P}\left\{\alpha_{k}^{\varepsilon}(x) \leqslant u\right\}, \quad u \in \mathbf{R}, \quad x \in E .
$$

From now on we will suppose that for any fixed sequence $\left(z_{k}\right)$ in $E$, the sequence $\alpha_{k}^{\varepsilon}\left(z_{k}\right), k \geqslant 0$, consists of independent r.v.'s.

$\mathrm{R}$ e mark 1. Results derived below hold also in the Euclidean space $\mathbf{R}^{d}, d>1$.

$\mathrm{R}$ e $\mathrm{m}$ a r $\mathrm{k} 2$. In what follows, to avoid notational complication, we use the same symbol $\theta^{\varepsilon}$ to denote different negligible functions, i.e., $\theta^{\varepsilon} \rightarrow 0$ as $\varepsilon \rightarrow 0$.

3. Increment process. Let us define the class of functions $g \in C_{3}(\mathbf{R})$, characterized by the conditions: $g(u)$ is a real-valued bounded continuous function with $g(u) / u^{2} \rightarrow 0$ as $|u| \rightarrow 0$. This is a convergence-determining class [7].

Theorem 1. Let the following conditions hold.

1. The switching Markov jump process $x(t), t \geqslant 0$, is uniformly ergodic with the stationary distributions (4).

2. The family of random variables $\alpha_{k}^{\varepsilon}(x), k \geqslant 0, x \in E$, is uniformly square-integrable, i.e.,

$$
\sup _{\varepsilon>0} \sup _{x \in E} \int_{|u|>c} u^{2} \Phi_{x}^{\varepsilon}(d u) \longrightarrow 0 \quad \text { as } \quad c \rightarrow \infty .
$$


3. Approximation of mean value:

$$
\int_{\mathbf{R}} u \Phi_{x}^{\varepsilon}(d u)=\varepsilon\left[a(x)+\theta^{\varepsilon}(x)\right]
$$

and $\sup _{x \in E}|a(x)| \leqslant a<\infty$.

4. Poisson approximation condition:

$$
\int_{\mathbf{R}} g(u) \Phi_{x}^{\varepsilon}(d u)=\varepsilon\left[\Phi_{x}(g)+\theta_{x}^{\varepsilon}(g)\right], \quad g \in C_{3}(\mathbf{R}),
$$

and $\sup _{x \in E}\left|\Phi_{x}(g)\right| \leqslant \Phi(g)<\infty$.

5. Square-integrability condition:

$$
\sup _{x \in E} \int_{\mathbf{R}} u^{2} \Phi_{x}(d u)<+\infty
$$

where the measure $\Phi_{x}(d u)$ is defined by the relation (see [7])

$$
\Phi_{x}(g)=\int_{\mathbf{R}} g(u) \Phi_{x}(d u), \quad g \in C_{3}(\mathbf{R}) .
$$

The negligible terms $\theta^{\varepsilon}(x)$ and $\theta_{x}^{\varepsilon}(g)$ in the above conditions satisfy:

$$
\sup _{x \in E}\left|\theta^{\varepsilon}(x)\right| \rightarrow 0, \quad \sup _{x \in E}\left|\theta_{x}^{\varepsilon}(g)\right| \rightarrow 0, \quad \varepsilon \rightarrow 0 .
$$

Then the increment process (6) converges weakly to the compound Poisson process

$$
A_{0}(t):=\sum_{k=1}^{\nu_{0}(t)} \alpha_{k}^{0}+t q a_{0}, \quad t \geqslant 0
$$

The distribution function $\Phi^{0}(u)$ of the i.i.d. random variables $\alpha_{k}^{0}, k \geqslant 0$, is defined on the convergence-determining class $C_{3}(\mathbf{R})$ of functions $g$ by the relation

$$
\mathbf{E} g\left(\alpha_{k}^{0}\right)=\int_{\mathbf{R}} g(u) \Phi^{0}(d u)=\frac{\widehat{\Phi}(g)}{\widehat{\Phi}(1)}, \quad g \in C_{3}(\mathbf{R}),
$$

where

$$
\widehat{\Phi}(g):=\int_{E} \rho(d x) \Phi_{x}(g), \quad \widehat{\Phi}(1):=\int_{E} \rho(d x) \Phi_{x}(\mathbf{R}) .
$$

The counting Poisson process $\nu_{0}(t)$ is defined by the intensity

$$
q_{0}:=q \widehat{\Phi}(1)
$$

The drift parameter $a_{0}$ is defined by

$$
a_{0}=\widehat{a}-\widehat{\Phi}(1) \mathbf{E} \alpha_{1}^{0}, \quad \widehat{a}:=\int_{E} \rho(d x) a(x) .
$$


Corollary 1. The increment process (6) with a finite number of jump values

$$
\begin{aligned}
\mathbf{P}\left\{\alpha_{k}^{\varepsilon}(x)=a_{m}\right\} & =\varepsilon p_{m}(x), \quad 1 \leqslant m \leqslant M, \\
\mathbf{P}\left\{\alpha_{k}^{\varepsilon}(x)=\varepsilon a_{0}\right\} & =1-\varepsilon p_{0}(x), \\
p_{0}(x) & =\sum_{m=1}^{M} p_{m}(x),
\end{aligned}
$$

converges weakly to the compound Poisson process (8) determined by the distribution function of jumps:

$$
\begin{array}{rr}
\mathbf{P}\left\{\alpha_{k}^{0}=a_{m}\right\}=p_{m}^{0}, & 1 \leqslant m \leqslant M, \\
p_{m}^{0}=\frac{\widehat{p}_{m}}{\widehat{p}_{0}}, \quad \widehat{p}_{m}=\int_{E} \rho(d x) p_{m}(x), & 1 \leqslant m \leqslant M .
\end{array}
$$

The intensity of the counting Poisson process $\nu_{0}(t), t \geqslant 0$, is defined by

$$
q_{0}:=q \widehat{p}_{0},
$$

the drift parameter $a_{0}$ is given in (12).

4. Increment process in an asymptotic split phase space. Now the switching Markov process $x^{\varepsilon}(t), t \geqslant 0$, is considered in the series scheme with a small series parameter $\varepsilon>0$, on an asymptotic split phase space

$$
E=\bigcup_{v \in V} E_{v}, \quad E_{v} \cap E_{v^{\prime}}=\varnothing, \quad v \neq v^{\prime},
$$

where $(V, \mathscr{V})$ is a compact measurable space. The case where $V$ is a finite set is of particular interest in applications.

The generator is given by the relation

$$
Q^{\varepsilon} \varphi(x)=\int_{E} Q_{\varepsilon}(x, d y)[\varphi(y)-\varphi(x)] .
$$

The transition kernel $Q_{\varepsilon}$ has the following representation:

$$
Q_{\varepsilon}(x, B)=q(x) P^{\varepsilon}(x, B)=Q(x, B)+\varepsilon Q_{1}(x, B),
$$

with the stochastic kernel $P^{\varepsilon}$ representation

$$
P^{\varepsilon}(x, B)=P(x, B)+\varepsilon P_{1}(x, B) .
$$

The stochastic kernel $P(x, B)$ is coordinated with the split phase space (16) as follows:

$$
P\left(x, E_{v}\right)=1_{v}(x):= \begin{cases}1, & x \in E_{v}, \\ 0, & x \notin E_{v} .\end{cases}
$$

In the sequel we suppose that the kernel $P_{1}$ is of bounded variation, i.e.,

$$
\left|P_{1}\right|(x, E)<+\infty \text {. }
$$


According to (19) and (20), the Markov process $x^{\varepsilon}(t), t \geqslant 0$, spends a long time in every class $E_{v}$ and the probability of transition from one class to another is $O(\varepsilon)$.

The phase merging scheme [9] is realized under the condition that the support Markov process $x^{0}(t), t \geqslant 0$, with the generator (2) is uniformly ergodic in every class $E_{v}, v \in V$, with the stationary distributions

$$
\pi_{v}(d x) q(x)=q_{v} \rho_{v}(d x), \quad q_{v}:=\int_{E_{v}} \pi_{v}(d x) q(x) .
$$

Let us define the merging function

$$
v(x)=v, \quad x \in E_{v} .
$$

By the phase merging scheme [9], the merged Markov process converges weakly:

$$
v\left(x^{\varepsilon}\left(\frac{t}{\varepsilon}\right)\right) \Longrightarrow \widehat{x}(t) \quad \text { as } \quad \varepsilon \rightarrow 0
$$

to the merged Markov process $\widehat{x}(t), t \geqslant 0$, defined on the merged phase space $V$ by the generating kernel

$$
\widehat{Q}\left(v, B_{\Gamma}\right)=\int_{E_{v}} \pi_{v}(d x) Q\left(x, B_{\Gamma}\right), \quad B_{\Gamma}=\bigcup_{v \in \Gamma} E_{v}, \quad \Gamma \in \mathscr{V} .
$$

The counting process of jumps, noted $\widehat{\nu}(t)$, can be obtained as the following limit (see [9]):

$$
\varepsilon \nu^{\varepsilon}\left(\frac{t}{\varepsilon}\right) \Longrightarrow \widehat{\nu}(t) \quad \text { as } \quad \varepsilon \rightarrow 0 .
$$

Theorem 2. Under conditions 2-5 of Theorem 1, in the phase merging scheme the increment process with Markov switching in series scheme

$$
A_{\varepsilon}(t):=\sum_{k=1}^{\nu^{\varepsilon}(t / \varepsilon)} \alpha_{k}^{\varepsilon}\left(x_{k}^{\varepsilon}\right), \quad t \geqslant 0,
$$

converges weakly to the additive semimartingale $A_{0}(t), t \geqslant 0$, which is defined by its predictable characteristics,

$$
B(t)=\int_{0}^{t} b(\widehat{x}(s)) d s, \quad b(v)=q_{v} \widehat{a}(v), \quad \widehat{a}(v):=\int_{E_{v}} \rho_{v}(d x) a(x) ;
$$

the modified second characteristic $\widetilde{C}^{\varepsilon}$ converges to

$$
\widehat{C}(t)=\int_{0}^{t} \widehat{C}(\widehat{x}(s)) d s
$$

where

$$
\widehat{C}(v)=q_{v} \int_{E_{v}} \rho_{v}(d x) C_{0}(x), \quad v \in V, \quad \text { and } \quad C_{0}(x)=\int_{\mathbf{R}} u^{2} \Phi_{x}(d u) .
$$


The predictable measure is

$$
\nu_{t}(g)=\int_{0}^{t} \widetilde{\Phi}_{\hat{x}(s)}(g) d s, \quad \widetilde{\Phi}_{v}(g)=q_{v} \widehat{\Phi}_{v}(g)
$$

where

$$
\widehat{\Phi}_{v}(g):=\int_{E_{v}} \rho_{v}(d x) \Phi_{x}(g) .
$$

R e m a r k 3 . The semimartingale $A_{0}(t), t \geqslant 0$, with predictable characteristics (27)-(29), can be represented in the following form:

$$
A_{0}(t)=\int_{0}^{t} A^{0}(d s ; \widehat{x}(s))
$$

or, in the equivalent increment form

$$
A_{0}(t)=\sum_{k=1}^{\hat{\nu}(t)} A_{\hat{x}_{k-1}}^{0}\left(\hat{\theta}_{k}\right)+A_{\hat{x}(t)}^{0}(\widehat{\gamma}(t)) .
$$

The compound Poisson processes $A_{v}^{0}(t)$ are defined by the generators

$$
A(v) \varphi(u)=q_{v}^{0} \int_{\mathbf{R}}[\varphi(u+z)-\varphi(u)] \Phi_{v}(d z),
$$

and $\nu_{v}^{0}(t)$ are the counting Poisson processes characterized by the intensity $q_{v}^{0}=q_{v} \widehat{\Phi}_{v}(1)$. Or in the explicit form

$$
A_{v}^{0}(t)=\sum_{\ell=1}^{\nu_{v}^{0}(t)} \alpha_{v \ell}^{0}+t q_{v} a_{v}^{0}, \quad v \in V
$$

for fixed $v \in V, \alpha_{v \ell}^{0}, \ell \geqslant 1$, are i.i.d. r.v.'s with a common distribution function defined by the measure

$$
\Phi_{v}^{0}(g)=\frac{\widehat{\Phi}_{v}(g)}{\widehat{\Phi}_{v}(1)} .
$$

The drift parameter is given by

$$
a_{v}^{0}=\widehat{a}(v)-\widehat{\Phi}_{v}(1) \mathbf{E} \alpha_{v 1}^{0} .
$$

$\mathrm{R}$ e $\mathrm{m}$ a $\mathrm{rk}$ 4. In applications, the limit semimartingale (30) can be considered in the following form

$$
A_{0}(t)=\sum_{k=1}^{\hat{\nu}(t)} \hat{\theta}_{k} c\left(x_{k-1}\right)+\widehat{\gamma}(t) c(\widehat{x}(t))+\mu_{0}(t),
$$

where $\mu_{0}(t)$ is a martingale fluctuation. The predictable term in (32) is a linear deterministic drift between jumps of the merged switching Markov process $\widehat{x}(t), t \geqslant 0$. 
5. Proof of theorems. In this section we will prove Theorem 2 only, since Theorem 1 can be proved by the same way using the limit theorem VIII.2.18 in [7]. The weak convergence in the functional space $D_{E}[0, \infty)$, developed here is considered in the setting of [7] and [5].

The space $\bar{C}_{0}\left(\mathbf{R}^{d}\right)$ of real-valued, bounded, uniformly continuous functions $\varphi(u), u \in \mathbf{R}^{d}$, with compact support, is used as a convergence determining class (see [5, Proposition 4.4]). The space $\bar{C}_{0}^{k}\left(\mathbf{R}^{d}\right), 1 \leqslant k \leqslant \infty$, of $k$-th continuously differentiable functions with compact support is used as an algebra separating points in $\mathbf{R}^{d}$ (see [5, Section 4.4]).

Let $\mathbf{B}$ be a Banach space of measurable real-valued bounded functions defined on $E$, with the sup-norm $\|f\|:=\sup _{x \in E}|f(x)|$. Let us denote by $P$ the operator of transition probabilities on $\mathbf{B}$ defined by $\operatorname{Pf}(x)=$ $\int_{E} P(x, d y) f(y)$.

Let $\mathscr{F}_{t}^{x}=\sigma\{x(s), s \leqslant t\}, t \geqslant 0$, be the natural filtration of the stochastic process $x(t), t \geqslant 0$. Let us define also the filtration $\mathbf{F}^{\varepsilon}=\left(\mathscr{F}_{t}^{\varepsilon}, t \geqslant 0\right)$, $\mathscr{F}_{t}^{\varepsilon}=\sigma\left\{x^{\varepsilon}(s), \alpha_{k}^{\varepsilon}\left(x_{s}\right), s \leqslant t, k \leqslant \nu^{\varepsilon}(t)\right\}$, and the filtration $\left(\mathscr{F}_{n}^{\varepsilon}=\sigma\left\{x_{k}^{\varepsilon}\right.\right.$, $k \leqslant n\}, n \geqslant 0)$. We suppose that the stochastic processes $x^{\varepsilon}(t), t \geqslant 0$, are defined on the stochastic basis $\mathscr{B}^{\varepsilon}=\left(\Omega, \mathscr{F}^{\varepsilon},\left(\mathscr{F}_{t}^{\varepsilon}, t \geqslant 0\right), \mathbf{P}^{\varepsilon}\right)$ with sample paths in $D_{E}[0, \infty)$, and phase space $(E, \mathscr{E})$ which is a Polish space.

The following lemmas are needed for the proof of Theorem 2 .

Lemma 1. Under the assumptions of Theorem 1, the predictable characteristics $\left(B^{\varepsilon}(t), C^{\varepsilon}(t), \Phi^{\varepsilon}(t ; g)\right)$ of the semimartingale

$$
A_{\varepsilon}(t)=\sum_{k=1}^{\nu^{\varepsilon}(t / \varepsilon)} \alpha_{k}^{\varepsilon}\left(x_{k}^{\varepsilon}\right), \quad t \geqslant 0,
$$

are defined by the following relations:

$$
B^{\varepsilon}(t)=\varepsilon \sum_{k=1}^{\nu^{\varepsilon}(t / \varepsilon)} b\left(x_{k-1}^{\varepsilon}\right)+\theta^{\varepsilon}(t), \quad t \geqslant 0,
$$

where $b(y)=P a(y), y \in E$, and the predictable measure

$$
\Phi^{\varepsilon}(t ; g)=\varepsilon \sum_{k=1}^{\nu^{\varepsilon}(t / \varepsilon)} P \Phi_{x_{k-1}^{\varepsilon}}(g)+\theta^{\varepsilon}(t ; g), \quad t \geqslant 0 .
$$

The modified second characteristic is

$$
\widehat{C}^{\varepsilon}(t)=\varepsilon \sum_{k=1}^{\nu^{\varepsilon}(t / \varepsilon)} C\left(x_{k-1}^{\varepsilon}\right)+\theta^{\varepsilon}(t), \quad t \geqslant 0,
$$

where $C(x)=P C_{0}(x)$ (see (28)). The continuous part of the second predictable characteristics is $C_{c}^{\varepsilon}(t) \equiv 0$. 
The negligible terms satisfy the following asymptotic conditions for every finite $T>0$ :

$$
\begin{aligned}
& \sup _{0 \leqslant t \leqslant T}\left|\theta^{\varepsilon}(t)\right| \stackrel{\mathbf{P}}{\longrightarrow} 0 \quad \text { as } \quad \varepsilon \rightarrow 0, \\
& \sup _{0 \leqslant t \leqslant T}\left|\theta^{\varepsilon}(t ; g)\right| \stackrel{\mathbf{P}}{\longrightarrow} 0 \quad \text { as } \quad \varepsilon \rightarrow 0, \text { for every } g \in C_{3}(\mathbf{R}) .
\end{aligned}
$$

Pr o o f. Concerning the semimartingale (33) we have (see [7, Theorem II.3.11])

$$
B^{\varepsilon}(t)=\sum_{k=1}^{\nu^{\varepsilon}(t / \varepsilon)} \mathbf{E}\left[\alpha_{k}^{\varepsilon}\left(x_{k}^{\varepsilon}\right) \mid \mathscr{F}_{k-1}^{\varepsilon}\right]
$$

In particular

$$
\mathbf{E}\left[\alpha_{k}^{\varepsilon}\left(x_{k}^{\varepsilon}\right) \mid \mathscr{F}_{k-1}^{\varepsilon}\right]=\int_{E} P^{\varepsilon}\left(x_{k-1}^{\varepsilon}, d y\right) \mathbf{E} \alpha_{k}^{\varepsilon}(y) .
$$

By condition 3 of Theorem 1, we get

$$
\begin{aligned}
\mathbf{E}\left[\alpha_{k}^{\varepsilon}\left(x_{k}^{\varepsilon}\right) \mid \mathscr{F}_{k-1}^{\varepsilon}\right] & =\varepsilon \int_{E}\left[P\left(x_{k-1}^{\varepsilon}, d y\right)+\varepsilon P_{1}\left(x_{k-1}^{\varepsilon}, d y\right)\right]\left[a(y)+\theta^{\varepsilon}(y)\right] \\
& =\varepsilon\left[P a\left(x_{k-1}^{\varepsilon}\right)+\theta^{\varepsilon}\left(x_{k-1}^{\varepsilon}\right)\right] .
\end{aligned}
$$

Now, relation (38) becomes

$$
B^{\varepsilon}(t)=\varepsilon \sum_{k=1}^{\nu^{\varepsilon}(t / \varepsilon)} P a\left(x_{k-1}^{\varepsilon}\right)+\theta^{\varepsilon}(t)
$$

where $\theta^{\varepsilon}(t)=\varepsilon \sum_{k=1}^{\nu^{\varepsilon}(t / \varepsilon)} \theta^{\varepsilon}\left(x_{k-1}^{\varepsilon}\right)$, which is a negligible term satisfying (37).

In the same way, as in (38), we get

$$
\Phi^{\varepsilon}(t ; g)=\sum_{k=1}^{\nu^{\varepsilon}(t / \varepsilon)} \mathbf{E}\left[g\left(\alpha_{k}^{\varepsilon}\left(x_{k}^{\varepsilon}\right)\right) \mid \mathscr{F}_{k-1}^{\varepsilon}\right]=\sum_{k=1}^{\nu^{\varepsilon}(t / \varepsilon)} P \Phi_{x_{k-1}^{\varepsilon}}^{\varepsilon}(g),
$$

where, by condition 4 of Theorem 1, we have

$$
\Phi_{x}^{\varepsilon}(g)=\mathbf{E} g\left(\alpha_{k}^{\varepsilon}(x)\right)=\int_{\mathbf{R}} g(u) \Phi_{x}^{\varepsilon}(d u)=\varepsilon\left[\Phi_{x}(g)+\theta_{x}^{\varepsilon}(g)\right] .
$$

Thus the predictable measure $\Phi^{\varepsilon}(t ; g)$ is represented by (35).

By condition 5 of Theorem 1, the modified second characteristic is represented as follows (see [7]):

$$
\widehat{C}^{\varepsilon}(t)=\sum_{k=1}^{\nu^{\varepsilon}(t / \varepsilon)} \mathbf{E}\left[\left(\alpha_{k}^{\varepsilon}\left(x_{k}^{\varepsilon}\right)\right)^{2} \mid \mathscr{F}_{k-1}^{\varepsilon}\right]=\varepsilon \sum_{k=1}^{\nu^{\varepsilon}(t / \varepsilon)} C\left(x_{k-1}^{\varepsilon}\right)+\theta^{\varepsilon}(t) .
$$

In the following lemma we shall use $B^{\varepsilon}(t), t \geqslant 0$ without the negligible term given in (34). 
Lemma 2. The coupled Markov process

$$
B^{\varepsilon}(t)=\varepsilon \sum_{k=1}^{\nu^{\varepsilon}(t / \varepsilon)} b\left(x_{k-1}^{\varepsilon}\right), \quad x^{\varepsilon}\left(\frac{t}{\varepsilon}\right), \quad t \geqslant 0
$$

characterized by the martingale

$$
\varphi\left(B^{\varepsilon}(t), x^{\varepsilon}\left(\frac{t}{\varepsilon}\right)\right)-\int_{0}^{t} L^{\varepsilon} \varphi\left(B^{\varepsilon}(s), x^{\varepsilon}\left(\frac{s}{\varepsilon}\right)\right) d s=\mu_{t}^{\varepsilon}, \quad t \geqslant 0,
$$

with respect to the filtration $\left(\mathscr{F}_{t / \varepsilon}^{\varepsilon}, t \geqslant 0\right)$, where $\varphi \in \mathscr{D}\left(L^{\varepsilon}\right)$, is defined by the generator $L^{\varepsilon}$, which is represented in explicit form

$$
L^{\varepsilon} \varphi(u, x)=\varepsilon^{-1} Q+\left[Q_{1}+Q_{0} B^{\varepsilon}(x)\right] \varphi(u, x),
$$

and in asymptotic form

$$
L^{\varepsilon} \varphi(u, x)=\varepsilon^{-1} Q \varphi(u, x)+\left[Q_{1}+Q_{0} B(x)\right] \varphi(u, x)+Q_{0} \theta^{\varepsilon}(x) \varphi(u, x),
$$

where

$$
\begin{aligned}
Q \varphi(\cdot, x) & =q(x) \int_{E} P(x, d y)[\varphi(\cdot, y)-\varphi(\cdot, x)] \\
Q_{1} \varphi(\cdot, x) & =q(x) \int_{E} P_{1}(x, d y) \varphi(\cdot, y) \\
Q_{0} \varphi(\cdot, x) & =q(x) \int_{E} P(x, d y) \varphi(\cdot, y), \\
B^{\varepsilon}(x) \varphi(u, \cdot) & =\varepsilon^{-1}[\varphi(u+\varepsilon b(x), \cdot)-\varphi(u, \cdot)] \\
B(x) \varphi(u, \cdot) & =b(x) \varphi_{u}^{\prime}(u, \cdot), \\
\theta^{\varepsilon}(x) \varphi(u, \cdot) & =\varepsilon^{-1}\left[\varphi(u+\varepsilon b(x), \cdot)-\varphi(u, \cdot)-\varepsilon b(x) \varphi_{u}^{\prime}(u, \cdot)\right] .
\end{aligned}
$$

P r o o f. For the proof of this lemma see [9, Corollary 4.4, p. 96].

Lemma 3. If

$$
\mathbf{E}\left|B^{\varepsilon}(0)\right| \leqslant b<+\infty
$$

then the family of processes

$$
B^{\varepsilon}(t)=\varepsilon \sum_{k=1}^{\nu^{\varepsilon}(t / \varepsilon)} b\left(x_{k-1}^{\varepsilon}\right)+B^{\varepsilon}(0), \quad t \geqslant 0, \quad \varepsilon>0
$$

satisfies the compact containment condition (see [5])

$$
\lim _{\ell \rightarrow \infty} \sup _{\varepsilon>0} \mathbf{P}^{\varepsilon}\left\{\sup _{0 \leqslant t \leqslant T}\left|B^{\varepsilon}(t)\right| \geqslant \ell\right\}=0 .
$$

For the proof of Lemma 3, we need the following lemma. 
Lemma 4 (see [5, Lemma 3.2, p. 174]). Let $x(t), t \geqslant 0$, be a Markov process defined by the generator $L$ and $\mathscr{G}_{t} \supset \mathscr{F}_{t}^{x}$. Then for any fixed $\lambda \in \mathbf{R}$ and $\varphi \in \mathscr{D}(L)$

$$
e^{-\lambda t} \varphi(x(t))+\int_{0}^{t} e^{-\lambda s}[\lambda \varphi(x(s))-L \varphi(x(s))] d s
$$

is a $\left(\mathscr{G}_{t}\right)$-martingale.

Proof of Lem a 3 . Put

$$
p_{\ell}^{\varepsilon}=\mathbf{P}^{\varepsilon}\left\{\sup _{0 \leqslant t \leqslant T}\left|B^{\varepsilon}(t)\right| \geqslant \ell\right\} .
$$

Let $\varphi_{0}(u)=\sqrt{1+u^{2}}$, thus $\varphi_{0}^{\prime}(u)=u / \sqrt{1+u^{2}}$. Hence

$$
\left|\varphi_{0}^{\prime}(u)\right| \leqslant 2 \leqslant 2 \varphi_{0}(u), \quad\left|\varphi_{0}^{\prime \prime}(u)\right| \leqslant 2 \leqslant 2 \varphi_{0}(u), \quad u \in \mathbf{R} .
$$

By inequality (5), we estimate

$$
\begin{aligned}
L^{\varepsilon} \varphi_{0}(u) & =\varepsilon^{-1} Q \varphi_{0}(u)+\left[Q_{1}+Q_{0} B(x)\right] \varphi_{0}(u)+Q_{0} \theta^{\varepsilon}(x) \varphi_{0}(u) \\
& \leqslant 2 q_{1} b \varphi_{0}(u)+2 \varepsilon q_{1} b \varphi_{0}(u) \leqslant C_{a} \varphi_{0}(u) .
\end{aligned}
$$

Let us define also the stopping time $\tau_{\ell}^{\varepsilon}$ :

$$
\tau_{\ell}^{\varepsilon}=\left\{\begin{array}{l}
\inf \left\{t \in[0, T]:\left|B^{\varepsilon}(t)\right| \geqslant \ell\right\} \\
T \quad \text { if }\left|B^{\varepsilon}(t)\right|<\ell, \quad \text { for all } t \in[0, T] .
\end{array}\right.
$$

By Lemma 4, applied to a stopping time instead of a fixed time $t$, we have that

$$
e^{-C_{a} t \wedge \tau_{\ell}^{\varepsilon}} \varphi_{0}\left(B^{\varepsilon}\left(t \wedge \tau_{\ell}^{\varepsilon}\right)\right)+\int_{0}^{t \wedge \tau_{\ell}^{\varepsilon}} e^{-C_{a} s}\left[C_{a} \varphi_{0}\left(B^{\varepsilon}(s)\right)-L^{\varepsilon} \varphi_{0}\left(B^{\varepsilon}(s)\right)\right] d s
$$

is a martingale. Due to (54) we get, for $s \leqslant t \wedge \tau_{\ell}^{\varepsilon}$,

$$
C_{a} \varphi_{0}\left(B^{\varepsilon}(s)\right)-L^{\varepsilon} \varphi_{0}\left(B^{\varepsilon}(s)\right) \geqslant 0,
$$

and from (56) we obtain

$$
\mathbf{E}\left[e^{-C_{a} t \wedge \tau_{\ell}^{\varepsilon}} \varphi_{0}\left(B^{\varepsilon}\left(t \wedge \tau_{\ell}^{\varepsilon}\right)\right)\right] \leqslant \mathbf{E} \mu_{t}^{\varepsilon}=\mathbf{E} \mu_{0}^{\varepsilon}=\mathbf{E} \varphi_{0}\left(B^{\varepsilon}(0)\right) .
$$

The convexity property of $\varphi_{0}$ and the inequality $\varphi_{0}(u) \geqslant 1$ provide the estimation

$$
p_{\ell}^{\varepsilon} \leqslant \mathbf{P}^{\varepsilon}\left\{\varphi_{0}\left(B^{\varepsilon}\left(\tau_{\ell}^{\varepsilon}\right)\right) \geqslant \varphi_{0}(\ell)\right\},
$$

and the Chebyshev inequality yields

$$
p_{\ell}^{\varepsilon} \leqslant \mathbf{E}\left[\varphi_{0}\left(B^{\varepsilon}\left(\tau_{\ell}^{\varepsilon}\right)\right)\right]\left(\varphi_{0}(\ell)\right)^{-1} .
$$

From inequality (59) together with inequality $e^{-\tau_{\ell}^{\varepsilon}} \geqslant e^{-T}$ (since $\tau_{\ell}^{\varepsilon} \leqslant T$ ), we obtain

$$
p_{\ell}^{\varepsilon} \leqslant e^{C_{a} T} \mathbf{E}\left[e^{-C_{a} \tau_{\ell}^{\varepsilon}} \varphi_{0}\left(B^{\varepsilon}\left(\tau_{\ell}^{\varepsilon}\right)\right)\right]\left(\varphi_{0}(\ell)\right)^{-1},
$$


and from (58) we get

$$
p_{\ell}^{\varepsilon} \leqslant e^{C_{a} T} \mathbf{E}\left[\varphi_{0}\left(B^{\varepsilon}(0)\right)\right]\left(\varphi_{0}(\ell)\right)^{-1} .
$$

Now, by the inequalities $\sqrt{1+u^{2}} \leqslant 1+|u|$ and (50), we get

$$
p_{\ell}^{\varepsilon} \leqslant e^{C_{a} T}(b+1)\left(\varphi_{0}(\ell)\right)^{-1} \longrightarrow 0 \text { as } \ell \rightarrow \infty .
$$

Lemma 5. The family of predictable characteristics $\left(B^{\varepsilon}(t), t \geqslant 0\right.$, $\varepsilon>0)$ is tight in $D_{\mathbf{R}}[0, T]$, for all $T>0$.

P r o o f. The coupled Markov process

$$
B_{0}^{\varepsilon}(t)=\varepsilon \sum_{k=1}^{\nu^{\varepsilon}(t / \varepsilon)} b\left(x_{k-1}^{\varepsilon}\right), \quad x^{\varepsilon}\left(t \varepsilon^{-1}\right), \quad t \geqslant 0,
$$

is defined by the generator (see Lemma 2)

$$
L^{\varepsilon} \varphi(x)=\varepsilon^{-1} Q \varphi(x)+\left[Q_{1}+Q_{0} B^{\varepsilon}(x)\right] \varphi(u, x) .
$$

For every nonnegative function $\varphi \in \bar{C}_{0}^{\infty}$ (R) we have $Q \varphi(u)=0$. Hence

$$
L^{\varepsilon} \varphi(u)=Q_{1} \varphi(u)+Q_{0} B^{\varepsilon}(x) \varphi(u),
$$

or, in explicit form,

$$
L^{\varepsilon} \varphi(u)=Q_{1} \varphi(u)+q(x) \int_{E} P(x, d y) \varepsilon^{-1}[\varphi(u+\varepsilon b(y))-\varphi(u)] .
$$

By assumptions (5) and (21), we get

$$
\varepsilon^{-1}|\varphi(u+\varepsilon b(y))-\varphi(u)| \leqslant b(y) \sup _{u}\left|\varphi^{\prime}(u)\right| \leqslant b C_{\varphi},
$$

and $Q_{1} \varphi(u) \leqslant q_{1} \varphi(u)$, where $|b(y)| \leqslant b<+\infty$ and $\left|\varphi^{\prime}(u)\right| \leqslant C_{\varphi}$.

So, the following estimation holds:

$$
\left|L^{\varepsilon} \varphi(u)\right| \leqslant C_{\varphi}
$$

where the constant $C_{\varphi}$ depends on the norms of $q(x), b(x)$, and $\varphi^{\prime}(u)$ but not on $\varepsilon$ nor on shifts of $\varphi[5],[18]$.

Now the process

$$
\eta^{\varepsilon}(t)=\varphi\left(B^{\varepsilon}(t)\right)+C_{\varphi} t, \quad t \geqslant 0,
$$

is a nonnegative $\left(\mathscr{F}_{t}^{\varepsilon}\right)$-submartingale for every real-valued nonnegative function $\varphi \in \bar{C}_{0}^{\infty}(\mathbf{R})$.

To see this, let us use the martingale characterization of coupled Markov process (63) for $t^{\prime}<t$,

$$
\begin{aligned}
\mathrm{E}\left[\eta^{\varepsilon}(t) \mid \mathscr{F}_{t^{\prime}}^{\varepsilon}\right]= & \mathbf{E}\left[\varphi\left(B^{\varepsilon}(t)\right) \mid \mathscr{F}_{t^{\prime}}^{\varepsilon}\right]+C_{\varphi} t \\
= & \mathbf{E}\left[\int_{0}^{t} L^{\varepsilon} \varphi\left(B^{\varepsilon}(s)\right) d s \mid \mathscr{F}_{t^{\prime}}^{\varepsilon}\right]+C_{\varphi} t+\mu_{t^{\prime}}^{\varepsilon} \\
= & \int_{0}^{t^{\prime}} L^{\varepsilon} \varphi\left(B^{\varepsilon}(s)\right) d s+C_{\varphi} t^{\prime}+\mu_{t^{\prime}}^{\varepsilon} \\
& +\mathbf{E}\left[\int_{t^{\prime}}^{t}\left(L^{\varepsilon} \varphi\left(B^{\varepsilon}(s)\right)+C_{\varphi}\right) d s \mid \mathscr{F}_{t^{\prime}}^{\varepsilon}\right] .
\end{aligned}
$$


So, the following relation takes place:

$$
\mathbf{E}\left[\eta^{\varepsilon}(t) \mid \mathscr{F}_{t^{\prime}}^{\varepsilon}\right]=\eta^{\varepsilon}\left(t^{\prime}\right)+\mathbf{E}\left[\int_{0}^{t}\left(L^{\varepsilon} \varphi\left(B^{\varepsilon}(s)\right)+C_{\varphi}\right) d s \mid \mathscr{F}_{t^{\prime}}^{\varepsilon}\right]
$$

Due to (67), the second term of the right-hand side is a nonnegative term. Hence

$$
\mathbf{E}\left[\eta^{\varepsilon}(t) \mid \mathscr{F}_{t^{\prime}}^{\varepsilon}\right] \geqslant \eta^{\varepsilon}\left(t^{\prime}\right), \quad \text { for } \quad t^{\prime}<t .
$$

Now, by (71) and Lemma 3, the family of predictable characteristics $\left(B^{\varepsilon}(t), t \geqslant 0, \varepsilon>0\right)$ satisfies the Stroock-Varadhan criteria of tightness [18], which holds in $D[0, \infty)$ too (see [19]).

An easy calculus gives us the following result.

Lemma 6. The generator $L$ of the limit coupled Markov process $B(t)=$ $\int_{0}^{t} b(\widehat{x}(s)) d s, \widehat{x}(t), t \geqslant 0$, is given by

$$
L \varphi(u, v)=\widehat{b}(v) \varphi_{u}^{\prime}(u, v)+\widehat{Q}_{1} \varphi(u, v)
$$

where $\widehat{b}(v)=q_{v} \int_{E_{v}} \rho_{v}(d x) b(x)=q_{v} \int_{E_{v}} \rho_{v}(d x) a(x)=q_{v} \widehat{a}(v)$.

Let us now fix the setting for the convergence theorem which will be used for proving the limit theorems 1 and 2 .

Consider a family of switching Markov processes

$$
\zeta^{\varepsilon}(t), \quad x^{\varepsilon}\left(t \varepsilon^{-1}\right), \quad t \geqslant 0,
$$

in the series scheme with a small series parameter $\varepsilon>0$, on the product space $\mathbf{R}^{d} \times E$, defined by the generators $L^{\varepsilon}, \varepsilon>0$. The domain of definition $\mathscr{D}\left(L^{\varepsilon}\right)$ is supposed to be dense in the space $\bar{C}\left(\mathbf{R}^{d} \times E\right)$ of real-valued, bounded, continuous functions $\varphi(u, x), u \in \mathbf{R}^{d}, x \in E$, with sup-norm $\|\varphi\|=\sup _{u \in \mathbf{R}^{d}, x \in E}|\varphi(u, x)|$.

The first switched component $\zeta^{\varepsilon}(t)$ takes values in the Euclidean space $\mathbf{R}^{d}, d \geqslant 1$. The second switching Markov process $x^{\varepsilon}(t / \varepsilon)$ is defined on the Polish space $(E, \mathscr{E})$ by the generator

$$
Q^{\varepsilon} \varphi(x)=\int_{E} Q^{\varepsilon}(x, d y)[\varphi(y)-\varphi(x)]
$$

in perturbed form with the kernel

$$
Q^{\varepsilon}(x, d y)=q(x)\left[P(x, d y)+\varepsilon P_{1}(x, d y)\right]=Q_{0}(x, d y)+\varepsilon Q_{1}(x, d y) .
$$

The generator of the coupled Markov process (73) is represented in the following singular perturbed form:

$$
L^{\varepsilon} \varphi(u, x)=\varepsilon^{-1}\left[Q^{\varepsilon}+Q_{1}^{\varepsilon}\right] \varphi(u, x),
$$

where the operator $Q_{1}^{\varepsilon}$ corresponds to the first switched component in (73). 
The switched Markov process $x^{\varepsilon}(t), t \geqslant 0$, is considered on the asymptotic split phase space (16). The merged phase space $V$ is defined by the merging function (23).

The limit Markov process

$$
\zeta(t), \widehat{x}(t), \quad t \geqslant 0
$$

is considered on the product space $\mathbf{R}^{d} \times V$ and is defined by the generator $L$, with domain $\mathscr{D}(L)$ dense in $\bar{C}\left(\mathbf{R}^{d} \times V\right)$.

The following convergence theorem is an adaptation of Theorem $8.10 \mathrm{in}$ [5, p. 234] to our conditions.

Theorem A. Suppose that the generator $L$ of the coupled Markov process $\zeta(t), \widehat{x}(t), t \geqslant 0$, on the phase space $\mathbf{R}^{d} \times V$ has at most one solution of a martingale problem in $D[0, \infty)$, and the closure of the domain $\mathscr{D}(L)$ contains a separating algebra $C_{a}$.

Suppose the family of Markov processes $\left(\zeta^{\varepsilon}(t), x^{\varepsilon}(t), t \geqslant 0, \varepsilon>0\right)$ on $\mathbf{R}^{d} \times E$ defined by the generators $L^{\varepsilon}, \varepsilon>0$, with domains $\mathscr{D}\left(L^{\varepsilon}\right)$ dense in $\bar{C}\left(\mathbf{R}^{d} \times E\right)$, satisfies the following conditions: that

(C1) there exists a collection of functions $\varphi^{\varepsilon}(u, x) \in \bar{C}\left(\mathbf{R}^{d} \times E\right)$ such

$$
\lim _{\varepsilon \rightarrow 0} \varphi^{\varepsilon}(u, x)=\varphi(u, v(x)) \in \bar{C}\left(\mathbf{R}^{d} \times V\right)
$$

and for every $T>0$

$$
\lim _{\varepsilon \rightarrow 0} \sup _{0 \leqslant t \leqslant T} \mathbf{E}\left|\varphi^{\varepsilon}\left(u, x^{\varepsilon}(t)\right)\right|<+\infty ;
$$

(C2) the uniform convergence of generators

$$
\lim _{\varepsilon \rightarrow 0} L^{\varepsilon} \varphi^{\varepsilon}(u, x)=L \varphi(u, v(x)),
$$

takes place, and $L^{\varepsilon} \varphi^{\varepsilon}, \varepsilon>0$, are uniformly bounded, $L \varphi \in \bar{C}\left(\mathbf{R}^{d} \times V\right)$;

(C3) the family of probability measures $\left(P^{\varepsilon}, \varepsilon>0\right)$ corresponding to the switching merged processes $\left(\zeta^{\varepsilon}(t), v\left(x^{\varepsilon}(t)\right), t \geqslant 0, \varepsilon>0\right)$ is relatively compact;

(C4) the convergence in probability of the initial values holds, i.e.,

$$
\left(\zeta^{\varepsilon}(0), x^{\varepsilon}(0)\right) \stackrel{\mathbf{P}}{\longrightarrow}(\zeta(0), \widehat{x}(0)) \quad \text { as } \varepsilon \rightarrow 0,
$$

with uniformly bounded expectation $\sup _{\varepsilon>0} \mathbf{E}\left|\zeta^{\varepsilon}(0)\right| \leqslant c<+\infty$.

Then the weak convergence in $D_{\mathbf{R}^{d} \times V}[0, \infty)$

$$
\left(\zeta^{\varepsilon}(t), v\left(x^{\varepsilon}(t)\right)\right) \Longrightarrow(\zeta(t), \widehat{x}(t)) \quad \text { as } \quad \varepsilon \rightarrow 0
$$

takes place.

$\mathrm{R}$ e $\mathrm{m}$ a r k 5. The main algorithmic conditions (8.53) and (8.54) of Theorem 8.10 in [5] are represented in conditions (78) and (80) of Theorem A. Additional conditions of boundedness (8.51) and (8.52) correspond 
to additional conditions in Conditions (C1) and (C2). All other conditions of Theorem 8.10 are represented in convergence theorem A in the same form.

$\mathrm{Pr}$ o of of $\mathrm{Th}$ e or e $\mathrm{m}$ 2. The coupled Markov processes

$$
B^{\varepsilon}(t), \quad x^{\varepsilon}\left(t \varepsilon^{-1}\right), \quad t \geqslant 0, \quad \varepsilon>0,
$$

in the series scheme are defined by the generators (42) in Lemma 2. For $\varphi(u, v(x)) \in \bar{C}_{0}^{2}(\mathbf{R} \times E)$ the generator (42) is represented in the asymptotic form

$$
L^{\varepsilon} \varphi=\varepsilon^{-1} Q \varphi+\left[Q_{1}+Q_{0} B(x)\right] \varphi+Q_{0} \theta^{\varepsilon}(x) \varphi,
$$

where, for $\varphi(u, \cdot)=\varphi(u)$,

$$
B(x) \varphi(u)=b(x) \varphi^{\prime}(u)
$$

and the residual operator

$$
\theta^{\varepsilon}(x) \varphi(u)=\varepsilon^{-1}\left[\varphi(u+\varepsilon b(x))-\varphi(u)-\varepsilon b(x) \varphi^{\prime}(u)\right] .
$$

For $\varphi(u) \in \bar{C}_{0}^{2}(\mathbf{R})$ the following negligibility condition holds

$$
\left|\theta^{\varepsilon}(x) \varphi(u)\right| \leqslant \varepsilon b\left\|\varphi^{\prime \prime}\right\| \longrightarrow 0 \quad \text { as } \varepsilon \rightarrow 0 .
$$

We will use a solution for the following singular perturbation problem (see [9, Lemma 3.3]):

$$
L^{\varepsilon} \varphi^{\varepsilon}=L \widehat{\varphi}+\varepsilon \theta^{\varepsilon}
$$

for the test functions

$$
\varphi^{\varepsilon}(u, x)=\varphi(u, v(x))+\varepsilon \varphi_{1}(u, x),
$$

where $\widehat{\varphi}=\hat{\varphi}(u, v) \in \bar{C}_{0}^{2}(\mathbf{R} \times V)$.

As the supporting Markov process $x(t), t \geqslant 0$, is uniformly ergodic, the bounded operator $Q$ on the Banach space $\mathbf{B}$ is reducible-invertible. Let $\Pi$ be the projector on the null space $N_{Q}=\{\varphi: Q \varphi=0\}, \operatorname{dim} N_{Q} \geqslant 1$, and let $R_{0}$ be the potential operator of $Q$ defined by $R_{0}:=[Q+\Pi]^{-1}-\Pi$.

The limit operator $L$ is given by the contracted operator

$$
L \widehat{\varphi}=\left(Q_{1}+Q_{0} B\right) \hat{\varphi} \hat{\varphi} .
$$

Consequently,

$$
L=\widehat{Q}_{1}+\widehat{Q_{0} B}
$$

where the contracted operators $\widehat{Q}_{1}$ and $\widehat{Q_{0} B}$ are defined by $\Pi Q_{1} \Pi=\widehat{Q}_{1} \Pi$ and $\widehat{Q_{0} B}(v) \varphi(u, v)=q_{v} a(v) \varphi_{u}^{\prime}(u, v)$, with $a(v):=\int_{E_{v}} \rho_{v}(d x) a(x)$.

The test function is realized by

$$
\begin{gathered}
\varphi \in N_{Q}, \\
\varphi_{1}(u, x)=R_{0}\left[L \widehat{\varphi}-\left(Q_{1}+Q_{0} B(x)\right)\right] \varphi,
\end{gathered}
$$


and

$$
\theta^{\varepsilon}(x)=\varepsilon\left[Q_{1}+Q_{0} B(x)\right] R_{0}\left[L \widehat{\varphi}-\left(Q_{1}+Q_{0} B(x)\right)\right] \varphi
$$

satisfies the negligibility condition (85).

The asymptotic representations (86), (87) and (88), (89) provide Conditions (C1) and (C2) of the convergence theorem A.

For the coupled Markov process (81) Condition (C3) is verified by Lemma 5.

The limit generator

$$
L \varphi(u, v)=\widehat{Q}_{1} \varphi(\cdot, v)+\widehat{b}(v) \varphi_{u}^{\prime}(u, \cdot)
$$

satisfies the preliminary conditions of Theorem A (see [19, Theorem 2]).

The limit generator $L$ given by relation $(72)$ is obtained by a singular perturbation approach.

Then, from Lemma 6 , the weak convergence in $D_{\mathbf{R} \times V}[0, \infty)$

$$
\left(B^{\varepsilon}(t), v\left(x^{\varepsilon}\left(t \varepsilon^{-1}\right)\right)\right) \Longrightarrow(B(t), \widehat{x}(t)) \quad \text { as } \quad \varepsilon \rightarrow 0
$$

takes place, where $B(t)=\int_{0}^{t} b(\widehat{x}(s)) d s$ is a stochastic process with continuous trajectories.

Since the process $B(t)$ has continuous trajectories (a.s.), the convergence in $D_{\mathbf{R}}[0, \infty)$ is equivalent to the uniform convergence in $C_{\mathbf{R}}[0, \infty)$ on every finite time interval $[0, T]$ in probability [2], i.e.,

$$
\sup _{0 \leqslant t \leqslant T}\left|B^{\varepsilon}(t)-B(t)\right| \stackrel{\mathbf{P}}{\longrightarrow} 0 \quad \text { as } \quad \varepsilon \rightarrow 0 \text {. }
$$

By the same way, we obtain the weak convergence in $D_{\mathbf{R}}[0, \infty)$ of the predictable measures, i.e., for any fixed function $g \in C_{3}(\mathbf{R})$,

$$
\Phi^{\varepsilon}(t ; g) \stackrel{\mathbf{P}}{\longrightarrow} \nu_{t}(g) \quad \text { as } \quad \varepsilon \rightarrow 0 .
$$

The modified second characteristic $\widetilde{C}^{\varepsilon}(t)$, given by $(36)$, converges to

$$
\widehat{C}(t)=\int_{0}^{t} \widehat{C}(\widehat{x}(s)) d s,
$$

as stated in Theorem 2 .

All the conditions of the limit theorem for semimartingales, Theorem IX.3.27, in [7] are fulfilled. Due to condition 2 of Theorem 1 in particular, the square integrability condition IX.3.24 in [7] holds true.

The strong majoration hypothesis is valid with the majoration function $F_{t}=t F$, with some constant $F$, due to the boundedness of all functions $b(x), C(x)$, and $\Phi_{x}(g)$ for $g \in C_{3}(\mathbf{R})$ and the known inequality [6]: $\varepsilon \nu^{\varepsilon}(t / \varepsilon) \leqslant c t, c>0$. Condition 5 of Theorem 1 implies the condition on big jumps for the last predictable measure of Theorem IX.3.27 in [7]. Conditions (iv) and (v) of Theorem IX.3.27 are obviously fulfilled. The weak 
convergence of predictable characteristics ([sup $\left.\beta-\mathbf{R}_{+}\right],\left[\gamma-\mathbf{R}_{+}\right]$, and $\left[\delta-\mathbf{R}_{+}\right]$ of Theorem IX.3.27 in [7]) is proved in Lemmas 1-6. The last condition 3.29 of Theorem IX.3.27 holds due to the uniform square-integrability condition 2 of Theorem 1 .

Hence, we get the weak convergence in $D_{\mathbf{R}}[0, \infty)$ of the increment process $A_{\varepsilon}(t)$ to the process $A_{0}(t)$ which is defined by the predictable characteristics (27)-(29), as stated in Theorem 2 above.

\section{REFERENCES}

1. Anisimov $V$. V. Switching processes: averaging principle, diffusion approximation and applications. - Acta Appl. Math., 1995, v. 40, № 2, p. 95-141.

2. Биллингсли П. Сходимость вероятностных мер. М.: Наука, 1977, 352 с.

3. Borovskikh Yu. V., Korolyuk V.S. Martingale Approximation. Utrecht: VSP, 1997, $322 \mathrm{p}$.

4. Cinlar E., Jacod J., Protter P., Sharpe M. J. Semimartingale and Markov processes. Z. Wahrscheinlichkeitstheor. verw. Geb., 1980, v. 54, № 2, p. 161-219.

5. Ethier S. N., Kurtz T. G. Markov Processes: Characterization and Convergence. New York: Wiley, 1986, $534 \mathrm{p}$.

6. Gut A. Stopped Random Walks. New York: Springer-Verlag, 1988, 199 p.

7. Жакод Ж., Ширяев А. Н. Пределіьные теоремы для случайных процессов. Т. 1, 2. М.: Физматлит, 1994, 544 с., 368 с.

8. Kluppelberg C., Mikosch T. Explosive Poisson shot noise processes with applications to risk reserves. - Bernoulli, 1995, v. 1, № 1-2, p. 125-142.

9. Korolyuk V.S., Korolyuk V.V. Stochastic Models of Systems. Dordrecht: Kluwer, $1999,185 \mathrm{p}$.

10. Korolyuk V.S., Limnios N. A singular perturbation approach for Liptser's functional limit theorem and some extensions. - Theory Probab. Math. Statist., 1998, № 58, p. $76-80$.

11. Korolyuk V.S., Limnios N. Diffusion approximation of integral functionals in merging and averaging scheme. - Theory Probab. Math. Statist., 1999, № 59, p. 101-107.

12. Korolyuk V.S., Limnios N. Diffusion approximation of integral functionals in double merging and averaging scheme. - Theory Probab. Math. Statist., 2000, №60, p. 8794.

13. Липчер P. Ш., Ширяев А.Н. Теория мартингалов. М.: Наука, 1986, 512 с.

14. Липчер P. ШI., Ширяев A.Н. Мартингалы и предельные теоремы для случайных процессов. - Итоги науки и техники, совр. проблемы матем., фундам. напр., 1989 , т. 45 , c. $159-253$.

15. Osaki S. Stochastic System Reliability Modeling. Singapore: World Scientific, 1985, $285 \mathrm{p}$.

16. Parzen E. Stochastic Processes. Philadelphia: SIAM, 1999, 324 p.

17. Prabhu N. U. Stochastic Storage Processes. New York, Berlin: Springer-Verlag, 1980, $140 \mathrm{p}$.

18. Stroock D. W., Varadhan S.R.S. Multidimensional Diffusion Processes. Berlin, New York: Springer-Verlag, 1979, 338 p.

19. Свириденко $M . H$. Мартингальная характеризация предельных распределений в пространстве функций без разрывов второго рода. - Матем. заметки, 1988, т. 43, № 5, p. 692-700. 